\title{
Developing the guidelines: the techniques of uniportal VATS for sublobar resection and middle lobectomy
}

\author{
Arthur Vieira ${ }^{1}$, Ricardo Oliveira ${ }^{2}$, Ivan Salgado de Azevedo ${ }^{3}$, Paula Ugalde Figueroa ${ }^{4}$ \\ ${ }^{1}$ Division of Thoracic Surgery, Vancouver General Hospital, Vancouver, BC, Canada; ${ }^{2}$ Division of Thoracic Surgery, Santa Casa de Misericórdia \\ da Bahia, Salvador, BA, Brazil; ${ }^{3}$ Division of Thoracic Surgery, Department of Oncology Oncobeda, Doctor Beda General Hospital, Rio de Janeiro, \\ Brazil; ${ }^{4}$ Division of Thoracic Surgery, Institut Universitaire de Cardiologie et de Pneumologie de Québec, Québec, QC, Canada \\ Correspondence to: Paula A. Ugalde, MD. Division of Thoracic Surgery, Institut Universitaire de Cardiologie et de Pneumologie de Québec, 2725 \\ Chemin Sainte-Foy, Ville de Québec, Québec, QC, Canada. Email: paula.ugalde@criucpq.ulval.ca.
}

Submitted Feb 01, 2019. Accepted for publication Apr 22, 2019.

doi: $10.21037 /$ jtd.2019.04.88

View this article at: http://dx.doi.org/10.21037/jtd.2019.04.88

\section{Introduction}

Since the landmark, randomized controlled trial by the Lung Cancer Study Group in 1995, lobectomy with systematic lymphadenectomy has been recognized as the standard treatment for early-stage, non-small cell lung cancer (NSCLC) (1). This study was done at a time where high definition computed tomography (CT) scan, positron emission tomography (PET) scan and endobronchial ultrasound (EBUS) were not available. Furthermore, a considerable portion of the procedures where wedge resections and all tumors were solid (the concept of GGO was still rudimental). These factors might have skewed the results resulting on the misleading concept that sublobar resection is not an adequate procedure for NSCLC.

Nowadays, the increased detection of early-stage NSCLC through modern thoracic imaging techniques and lung cancer screening programs $(2,3)$ has raised the question of whether there is a role for limited lung resections, such as wedge and segmentectomy, in the treatment of small tumors. Two multi-institutional, prospective, randomized trials are currently underway to elucidate if sublobar resection offers at least similar overall survival when compared with lobectomy for early-stage NSCLC $(4,5)$. Both have completed accrual and the results are expected by 2020 . These trials will have major impact in clinical practice.

Numerous non-randomized trials have analyzed the role of anatomical segmentectomy in the treatment of peripheral, stage IA NSCLC. An anatomical segmentectomy is favored over a lobectomy when the lesion is $<2 \mathrm{~cm}$, when the tissue margins are $>2 \mathrm{~cm}$ or greater than the tumor's diameter, and when the tumor is peripherally located. A sublobar resection is indicated for tumors with low maximum standardized uptake value (SUVmax) on PET-CT scan and in patients with poor pulmonary function or major comorbidities that contraindicate lobectomy (6-11).

Histologic patterns of lung adenocarcinoma classify tumors from least invasive, such as adenocarcinoma in situ (AIS) and minimally invasive adenocarcinomas (MIA), to most invasive, such as micropapillary and solid patterns (12). Ground glass opacities (GGOs) are a less invasive histologic type and a have favorable prognosis. Growing experience confirms not only a direct correlation between CT imaging of GGOs and histology (13) but also between histology and prognosis (14). These lesions might, therefore, be suitable to a lung-sparing strategy, primarily to avoid lobectomy and save parenchyma for future resections.

Minimally invasive thoracic surgery has become the standard approach to early-stage lung cancer either for lobectomy or sublobar resections. It requires precise movements, a profound knowledge of anatomy, and comfort with specialized equipment to avoid technical complications, especially vascular accidents. Some surgeons believe that it is safer and easier to perform lung surgery through multiple ports; however, there is no data that validates this impression. Because vision is the predominant sense used during a video-assisted thoracoscopic surgery (VATS) procedure, we believe that single-port (uniportal) technique offer a safer approach, because of the straight, frontal view of the pulmonary hilum elements facilitates a 


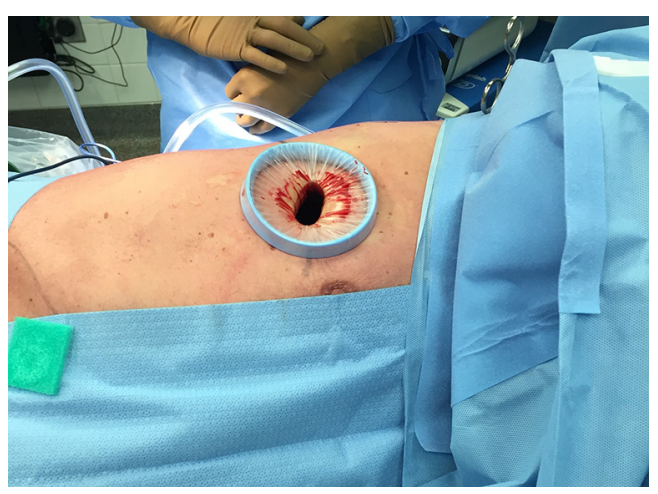

Figure 1 Uniportal incision in the $5^{\text {th }}$ right intercostal space with a wound protector.

safe dissection. For this reason, the uniportal approach has been our standard VATS approach since January 2014. In this article, we describe the technical aspects of common anatomical segmentectomies using a uniportal approach in an attempt to standardize the technical aspects of these procedures.

\section{Preoperative evaluation}

Based on recent guidelines, preoperative evaluation consists of two parts: a functional evaluation and an oncological evaluation. During the functional evaluation, the clinician gathers a full history and conducts a physical exam. A complete blood count (CBC), platelet count, and a blood chemistry profile are obtained. Pulmonary function tests (PFTs) and an electrocardiogram (ECG) are performed. Further testing may be indicated according to the patient's current physical status, previous medical history or abnormal PFTs.

To assess tumor oncology, the patient should be evaluated with a CT scan of the chest and a CT scan of the upper abdomen with contrast. They should undergo a PET scan, bronchoscopy, and ideally the tumor should be biopsy proven. Mediastinal staging, using EBUS or cervical mediastinoscopy, is indicated if there are PET-avid or enlarged ( $>1 \mathrm{~cm}$ in the shortest axis) lymph nodes.

A meticulous preoperative assessment of the chest CT is crucial to decide whether a sublobar resection might be appropriate. Segmentectomy should be considered in patients with lesions $<2 \mathrm{~cm}$ in the largest dimension. Understanding of the segmental anatomy, with special attention to identify each segmental artery, vein, and bronchus and its proximity to the lesion will assure

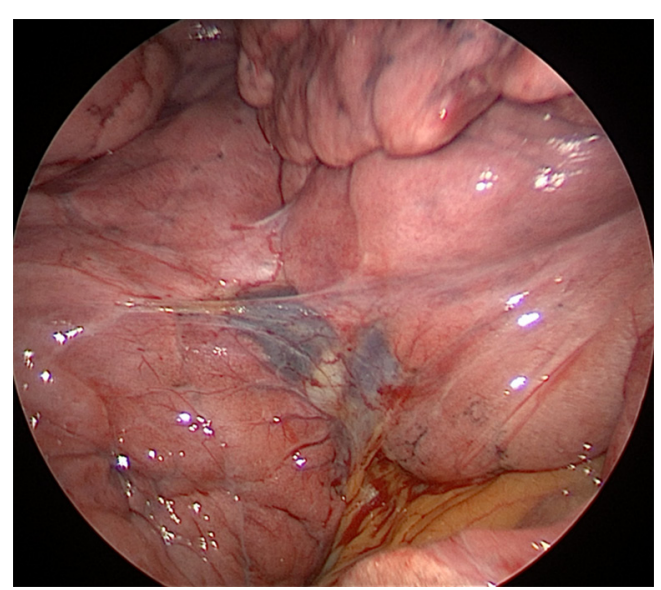

Figure 2 Anterior view through the incision in the $5^{\text {th }}$ right intercostal space.

adequate oncologic margins. For tumors located close to intersegmental planes, preoperative evaluation with CT scan measurements and perioperative assessment of tumor margins with frozen tissue samples will dictate the feasibility of the sublobar resection.

\section{Surgery}

Some technical principles are similar for all the segmentectomies. Under general anesthesia and one-lung ventilation with a double lumen endotracheal tube, the patient is placed in a lateral decubitus, with the hips flexed. The surgery is performed through a single port (uniportal) with a $3-4 \mathrm{~cm}$ incision at the $5^{\text {th }}$ intercostal space for the upper lobes and the right middle lobe and $6^{\text {th }}$ intercostal space for the lower lobes, in general at the anterior axillary line (Figure 1). A preemptive intercostal nerve block is routinely done at the incision site and in the $3^{\text {rd }}, 4^{\text {th }}$ and $5^{\text {th }}$ intercostal space for upper lobe procedures and $4^{\text {th }}, 5^{\text {th }}$ and $6^{\text {th }}$ intercostal spaces for lower lobe procedures. After transecting the serratus anterior muscle, a tunnel is created, with a finger using the rib as a rail, under the serratus toward the patient's back and is used for transection of the intercostal muscle with chest wall preservation. This internal thoracotomy promotes better exposure (Figure 2), more comfort during the procedure, and easier removal of the specimen from the cavity. A wound protector is used for optimal exposure and to facilitate instrumentation. Dissection and traction are then individualized based on the location of the tumor. 


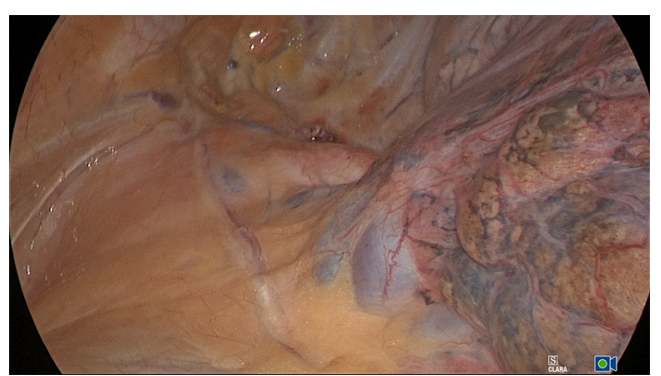

Figure 3 Left upper lobe (LUL) vein.

\section{Right upper lobe (RUL) posterior (S2) segmentectomy}

For a RUL posterior segmentectomy, traction is placed on the lung anteriorly to have a wide exposure of the posterior hilum. Dissection starts at the border between intermedius bronchus (IB) and the lower lobe and moves toward the upper lobe. This will expose the angle between bronchus take off for the RUL and the IB. The posterior portion of the greater fissure can be dissected with a stapler, ultrasonic scalpel, or a hook cautery, and this will help expose the pulmonary artery (PA). Lymphadenectomy is performed along the dissection to allow a safer and clearer exposure of the vessels and to confirm early-stage disease. Next, we position the RUL towards the apex of the chest to verticalize the superior segmental artery and vein. This exposure will allow us to see alignment of the vein, artery, and bronchus. The RUL posterior ascending arterial and venous branches are encircled and ligated with an energy device (for vessels measuring up to $7 \mathrm{~mm}$ ) (15) or with an endostapler and vascular load (for larger vessels). Subsequently, the posterior segment of the RUL is positioned vertically to facilitate the dissection and stapling of the corresponding bronchus. We routinely perform bronchoscopy at this time to confirm the segmental bronchus. The lung is slowly reexpanded to define the borders of the segment, or the distal bronchial stump may be lifted and used as a reference for stapling the parenchyma. The RUL posterior segment is retracted horizontally and towards the diaphragm. Finally, an intercostal nerve blockade is performed, the specimen is removed in a protective bag, followed by insertion of a 24-French chest drain and full re-expansion of the lung.

\section{Left upper lobe (LUL) trisegmentectomy (S1+2 + 53)}

For LUL trisegmentectomy $(\mathrm{S} 1+\mathrm{S} 2+\mathrm{S} 3)$, the dissection

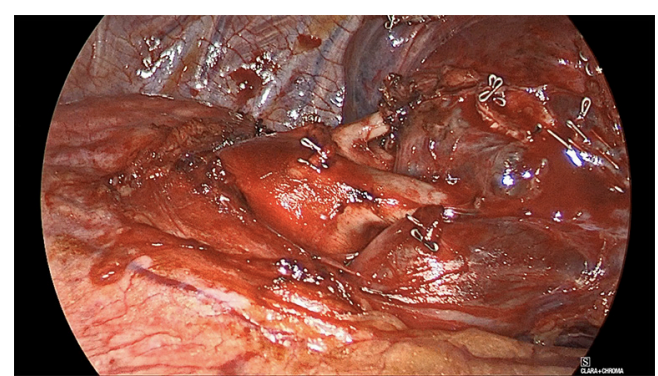

Figure 4 Tri-segment arterial branches.

initiates at the anterior hilum at the level of the LUL vein. Traction is placed on the LUL to identify the superior pulmonary vein and all its branches (Figure 3). This will pinpoint the angle where the apical and anterior branches should be dissected, while preserving the lingular vein. The first two veins are taken down with a stapler and a vascular load. The arterial branches are immediately lateral. The surgeon must be aware of the frequent vascular variations in the LUL lobe that result in remarkable differences in the number of arterial branches. Lymphadenectomy is performed along the dissection to allow a safer and clearer exposure of the vessels. By removing the lobar nodes, we gain optimal exposure to the apical branches of the PA. Next, the lung is retracted horizontally and inferiorly, towards the diaphragm. All arterial branches should be positioned vertically for safer dissection (Figure 4). Using a vascular load, the arterial branches are stapled en bloc or separately, from anterior to posterior, surrounding the PA towards the left lower lobe. Lastly, we proceed to bronchial dissection. The LUL is retracted vertically towards the lateral chest wall. We consider it essential to dissect the bronchus distally towards the parenchyma in order to identify segmental bronchial bifurcation, which is usually marked by a $12 \mathrm{~L}$ lymph node (Figure 5). After lymphadenectomy in this region, the lingular bronchus is identified and preserved, and the tri-segmental bronchus is exposed and ligated with an endostapler. To complete the anatomical resection, the lung is re-expanded by the anesthetist; the borders of the segment should be visible. The LUL is retracted superiorly towards the apex of the left chest. Another option is to lift the distal segment of the bronchus and use it as reference for stapling the parenchyma. Finally, an intercostal nerve blockade is performed (Figure 6), the specimen is removed in a protective bag, a 24-French chest drain is inserted, and the lung is fully re-expanded (Figure 7). 


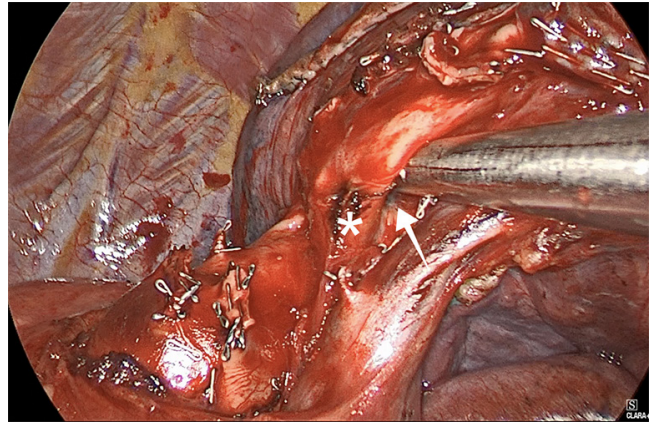

Figure 5 LUL bronchial bifurcation with the 12L lymph node (arrow) and the tri-segment bronchus (asterisk).

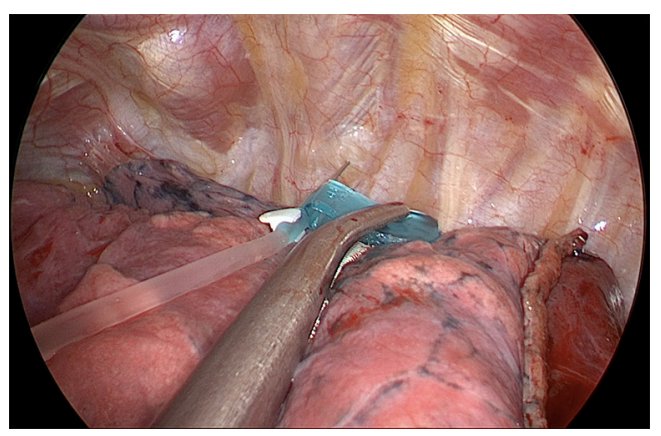

Figure 6 Intercostal nerve block before specimen removal.

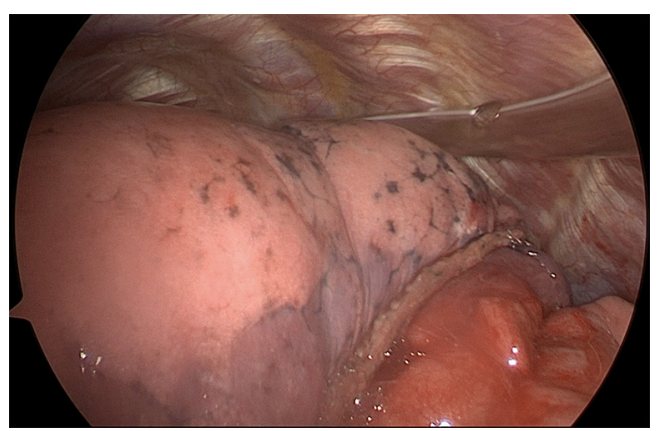

Figure 7 Chest tube inserted, and the lung being re-expanded.

\section{LUL lingulectomy}

For LUL lingulectomy, traction is placed on the LUL towards the spine, which provides good exposure of the anterior portion of the hilum. The mediastinal pleura is dissected between superior and inferior pulmonary vein. Dissection progresses towards the superior vein, and the branch of the lingular vein is easily identified (Figure 8). With the help of right-angle forceps, the vessel is encircled

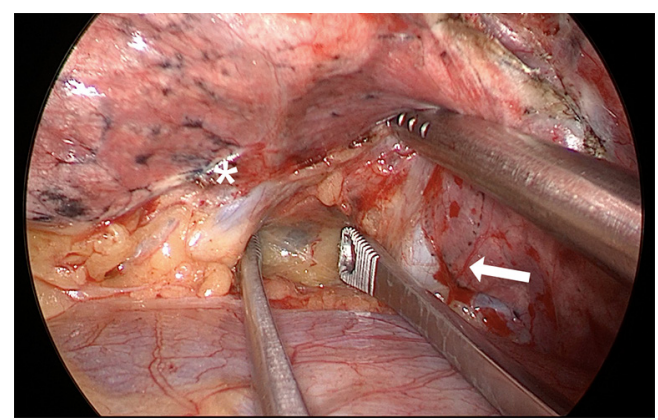

Figure 8 Dissection between the lingular vein (asterisk) and LLL vein (arrow). LLL, left lower lobe.

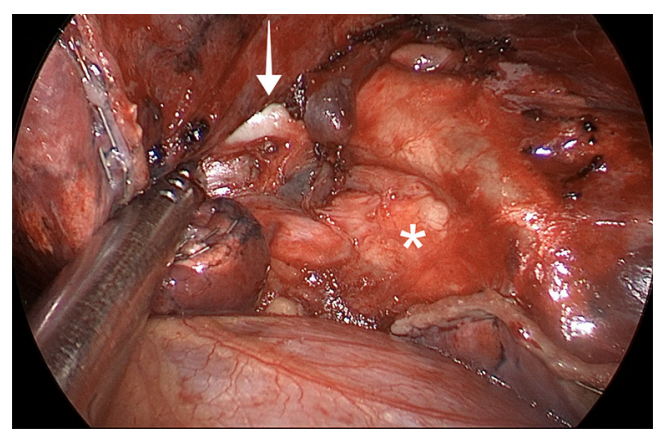

Figure 9 Dissection of lingular arteries (arrow). The bronchus is visible behind the PA (asterisk). PA, pulmonary artery.

and stapled with a vascular, $30-\mathrm{mm}$ load. It is also becoming common to use energy devices for the control of these small vascular branches. The lingular bronchus is just behind the vein. At this point, it is important to evaluate the fissure. In cases of complete or nearly complete fissure, we dissect towards the PA and identify the lingular branch (Figure 9). With a lung grasper, the parenchyma can be lifted such that the lingular artery and bronchus are in a more vertical position. The exposure is highly improved, and the dissection will be performed from a safer angle. If the fissure is incomplete, however, we use a peanut dissection sponge to dissect between the two pulmonary veins towards the bronchus. This maneuver will allow exposure of the interlobar carina and the PA. Then, we dissect the fissure with the stapler until we are close to the PA. At this point, we lift the parenchyma so the lingular artery and bronchus are in a vertical position and identify the pre-adventitial plane of the artery. With right-angle forceps, the artery can be encircled, and the vessel can be controlled with a stapler and vascular load or with an energy device. We ask the 


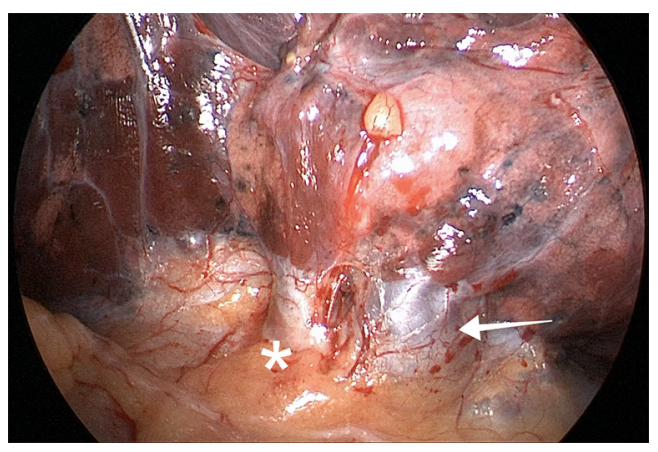

Figure 10 Anterior view of the hilum, showing exposure for a RML lobectomy, the RML vein (asterisk), and the RUL vein (arrow). RML, right middle lobectomy; RUL, right upper lobe.

anesthetist to introduce the bronchoscope in the lingular bronchus, so the transillumination helps to precisely identify the bronchial segment. In general, we do not ventilate the lung to complete the segmentectomy. Lymphadenectomy is performed along the dissection to allow a safer and clearer exposure of the vessels and to confirm early-stage disease.

\section{Right middle lobectomy (RML)}

When performing a RML, we position the right lung lobes posteriorly and use a hook cautery or an ultrasonic device to dissect the superior pulmonary vein and open the mediastinal pleura at the base of the RML vein (Figure 10). Using this maneuver, we can easily identify the venous branch for the middle lobe. It is essential to release the vein from the bronchus and adjacent attachments. The right lower lobe (RLL) and RML/RUL are lifted vertically towards the lateral chest wall, and the stapler is placed around the middle lobe vein towards the posterior hilum. Even though we don't see the tip of the stapler in this maneuver, if the lung is lifted as previously indicated, there is no risk of injuring structures behind the RML vein. The uniportal approach with a frontal view allows an excellent view of the interlobar PA within the fissure and the middle lobe bronchus behind the vein (Figure 11). We always look for the correct plane and an optimal dissection to allow proper mobility, which is crucial for safe stapler insertion. The use of curved-tip stapler technology facilitates placement around pulmonary vessels through a single incision. Next, we begin the dissection of the anterior portion of the major fissure, if it is incomplete. For this step, the RLL is moved towards the diaphragm, and the RML is grasped and directed towards the apex of the right side of the chest, which opens the major fissure. After

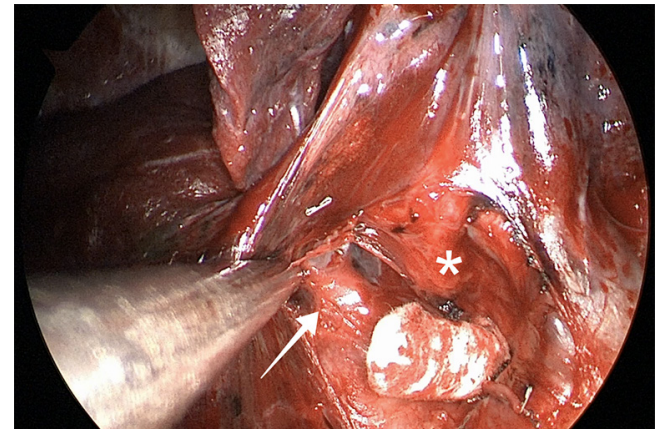

Figure 11 Bronchus (asterisk) and the arterial branches (arrow) to the RML. RML, right middle lobectomy.

dissection of the interlobar PA within the fissure, right-angle forceps are introduced in the space between the superior and inferior pulmonary veins. The tips of the instrument must come out anteriorly to the interlobar PA, which has already been dissected. Next, the anterior portion of the major fissure is divided, keeping the stapler blade away from the artery. Then, the dissection is concentrated on the RML bronchus using a hook cautery or blunt dissection with a peanut dissection sponge, while the RML is lifted towards the lateral chest wall. The lymph nodes between the vein, the bronchus, and the arterial branches should be dissected and removed systematically for a safe and oncologically sound procedure. The RML bronchus is then stapled.

After division of the vein and the bronchus, one or two arterial branches to the middle lobe are identified, dissected, and divided with a vascular load. With vertical traction of the lobe, it is frequently feasible to staple both middlelobe arteries with only one stapler load. Now, the middle lobe is attached to the upper lobe by only the minor fissure. The upper lobe vein and the stump of the RML bronchus provide landmarks for proper stapling (Figure 12). Gentle caudal traction is then applied to the middle lobe to place it in its anatomical position. It is advisable to have the interlobar PA well-dissected to permit free and safe passage of the stapler jaws. When the lobectomy is completed, the lobe is removed from the cavity using a protective bag, and a 24-French chest tube is placed for drainage.

\section{Superior (S6) segmentectomy}

For a superior segmentectomy (S6), we usually start by identifying the interlobar PA within the fissure. Whether the fissure is complete or not dictates our next actions. 


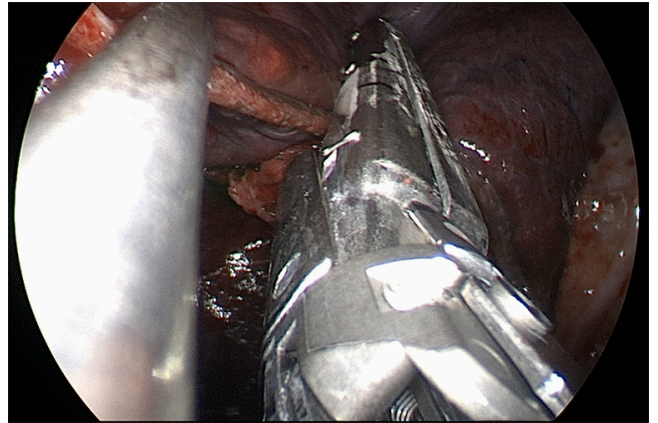

Figure 12 Minor fissure (between the RUL and RML) being divided. RML, right middle lobectomy; RUL, right upper lobe.

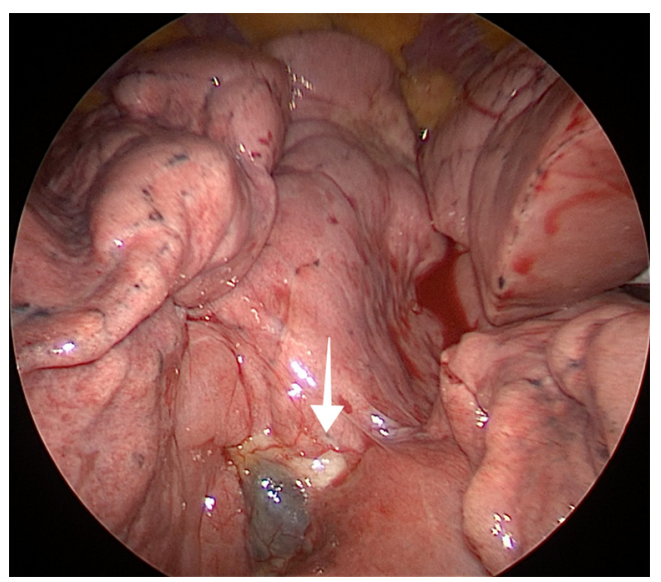

Figure 13 Complete major fissure with exposed PA (arrow). PA, pulmonary artery.

In cases with a complete fissure, the PA is easily seen and exposed after minimal dissection within the fissure (Figure 13). In cases of incomplete fissure, the PA can be identified in the fissure by dissecting from posterior to anterior or from anterior to posterior. By grasping and lifting the parenchyma of S6 segment and the posterior segment of the upper lobe, a "tunnel" just above the IB is created to identify the PA and, once a path is made, the fissure is progressively divided until we fully expose the PA (Figure 14). Otherwise, in less favorable conditions, where the fissure is totally incomplete, the interlobar PA may be visualized from anterior to posterior. After dissection between the superior and inferior pulmonary veins, a plane is developed superiorly towards the bronchial tree (Figure 15). This maneuver will expose the PA. Again, a tunnel is developed between the PA and the parenchyma, and the fissure is completely dissected with the stapler.

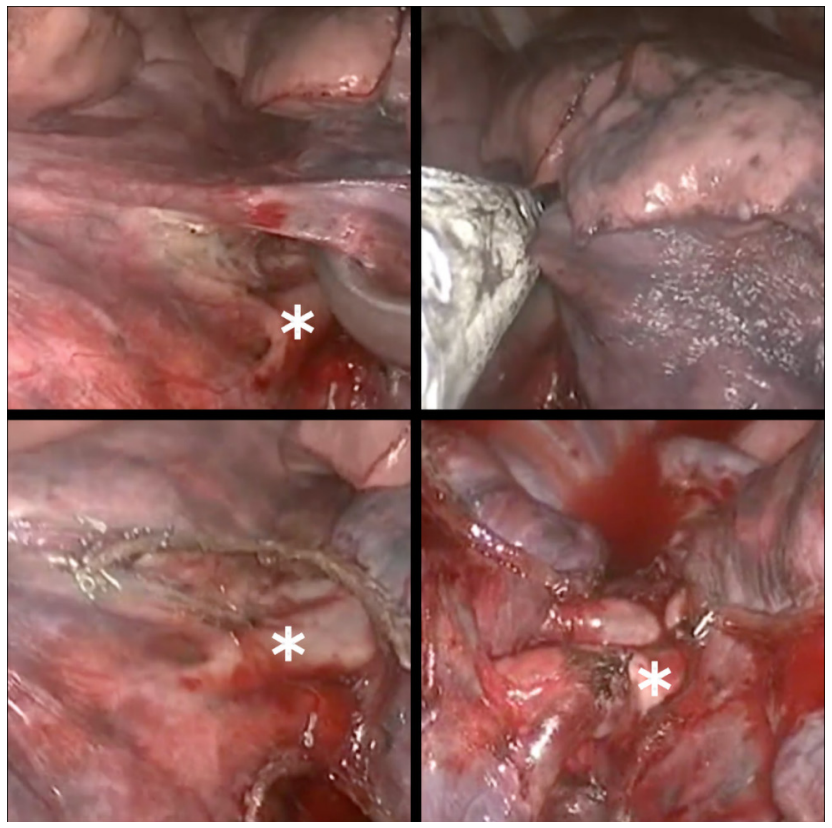

Figure 14 Creation of a tunnel above the pulmonary artery (asterisk) and division of an incomplete fissure.

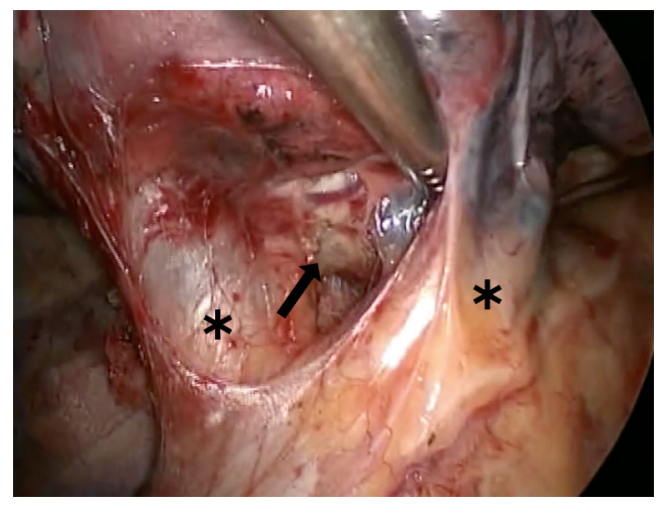

Figure 15 Dissection between pulmonary veins (asterisk) and lower lobe bronchus is exposed. The PA is found above the bronchus (arrow). PA, pulmonary artery.

Our objective is to isolate the superior segmental PA branch (A6) (Figure 16). This dissection is easier when the adventitial plane of the artery is identified and all lymph nodes are removed along the dissection. Commonly, one arterial branch to the superior segment (A6) is found, but, a second A6 branch is seen in $20 \%$ of patients (16). After A6 division, the superior segment of the bronchus (B6) is visualized (Figure 17). The superior segment should be grasped and lifted slightly towards the apex of the chest. 


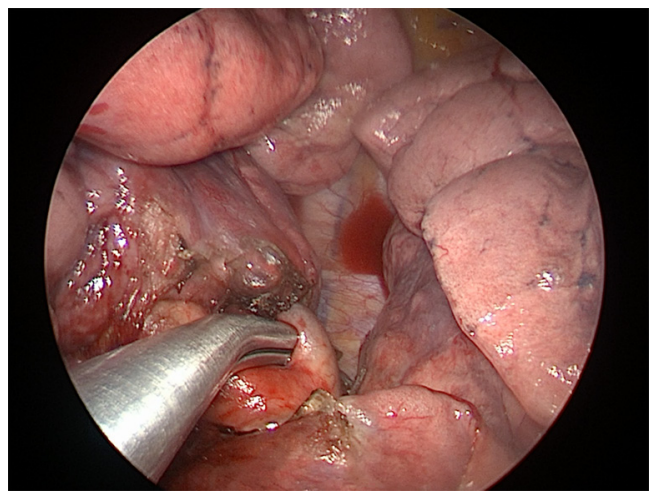

Figure 16 Superior segment artery (A6) individualized.

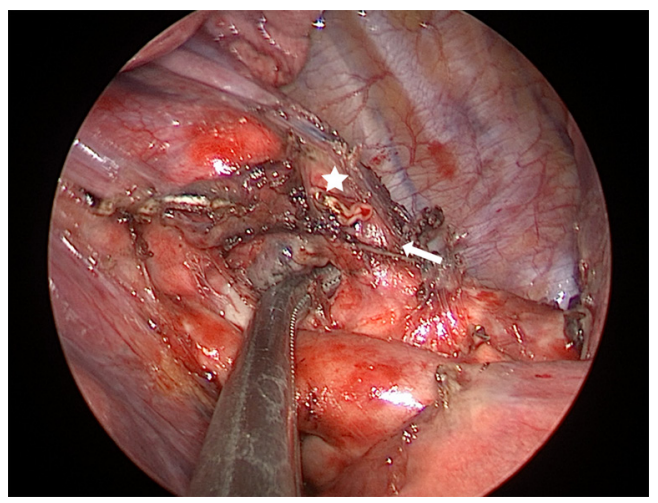

Figure 17 Dissection of the superior segment bronchus (arrow). The A6 stump (star) is seen distally.

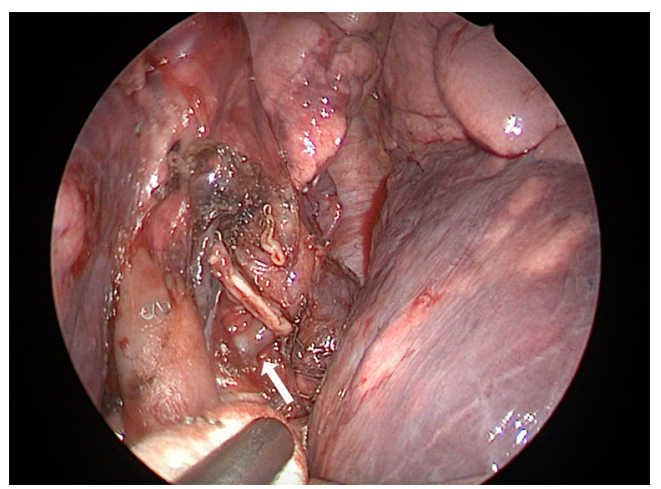

Figure 18 The superior segment vein (arrow) is identified after B6 division.

Again, removing lymph nodes might help at this point. Before B6 transection, we confirm lower lobe bronchial segmentation endoscopically to avoid erroneous stapling the basilar segments of the bronchi. Following B6 division, the superior segment vein (V6) is identified arising from the posterior mediastinum (Figure 18). V6 may be divided individually or en bloc with the parenchyma if oncologic margins are adequate. The superior segment is positioned gently towards the diaphragm. Finally, the parenchyma is sectioned, and the specimen is removed. Inflation/ deflation techniques and utilization of indocyanine-green (ICG) have been described to improve visualization of the intersegmental plane $(17,18)$. Intercostal nerve blockage is performed, and the specimen is removed in a protective bag. As with other procedures, the last step is the insertion of a 24-French chest tube for drainage.

\section{Basilar segmentectomy}

Basilar segmentectomies are interesting, because the vein, artery, and bronchial segment are all located towards the anterior part of the pulmonary hilum, so the complete procedure is performed with almost the same exposure. Depending on the fissure, dissection can be started with the artery or with the vein. With an incomplete fissure, we place a lung grasper lifting the segment, and the dissection starts at the inferior pulmonary ligament and proceeds all the way up until superior pulmonary vein. The path between both veins is dissected towards the aorta, which makes the inferior pulmonary vein more mobile. In general, the vein has only 2 branches, the superior segment and the basal pyramid. From the vein, using a peanut dissection sponge, it is possible to dissect the bronchus towards the interlobar carina. This will allow some visualization of the PA. Gently, the plane between the parenchyma and the PA is developed, so the fissure above the artery can be dissected with the stapler. The pyramid arterial branch is dissected and controlled with a vascular stapler. Next, the vein can be easily stapled, but care must be taken that the superior segment vein is being preserved. Lastly, the bronchus is encircled and stapled. With some traction on the bronchial and vein stump, we can better position the lung to complete the parenchymal segmentectomy.

If the fissure is complete, the lower lobe is placed towards the diaphragm, and the upper lobe is directed towards the apex of the chest exposing the major fissure. Our preferred approach is to begin with the interlobar PA dissection following same strategies and tips described above. Maintaining the same position, the basilar segment bronchus is dissected and divided subsequently. Finally, we grasp and retract the lower lobe superiorly and anteriorly allowing the pulmonary ligament to be sectioned and 
posterior mediastinal pleura to be opened. With the lung retracted anteriorly, the pulmonary vein to the basilar segments is dissected and divided while preserving the vein to the superior segment. For the parenchyma division, we use the vein as a landmark for the intersegmental plane. Lymphadenectomy according to current guidelines is always performed. Intercostal nerve blockage is administered, and the specimen is removed in a protective bag. Finally, a 24-French chest tube is inserted for drainage.

\section{Conclusions}

Uniportal anatomical segmentectomy requires comprehensive knowledge of thoracic anatomy and proven dexterity with minimally invasive surgical techniques. In this article, we describe the technical aspects of common anatomical segmentectomies using a uniportal approach with the hope of standardizing these advanced procedures. Although the indications for sublobar resections are still a matter of debate, thoracic surgeons should be involved in training programs to develop the necessary skills to adopt these techniques, as there will likely continue to be a need for sublobar resections.

\section{Acknowledgments}

None.

\section{Footnote}

Conflicts of Interest: The authors have no conflicts of interest to declare.

Informed Consent: Written informed consent was obtained from the patient for publication of this manuscript and any accompanying images.

\section{References}

1. Ginsberg RJ, Rubinstein LV. Randomized trial of lobectomy versus limited resection for T1 N0 non-small cell lung cancer. Lung Cancer Study Group. Ann Thorac Surg 1995;60:615-22; discussion 622-3.

2. Tammemagi MC, Schmidt H, Martel S, et al. Participant selection for lung cancer screening by risk modelling (the Pan-Canadian Early Detection of Lung Cancer [PanCan] study): a single-arm, prospective study. Lancet Oncol 2017;18:1523-31.
3. Dutch Belgian randomised lung cancer screening trial (NELSON). Available online: https://doi.org/10.1186/ ISRCTN63545820

4. Comparison of Different Types of Surgery in Treating Patients With Stage IA Non-Small Cell Lung Cancer. Available online: https://clinicaltrials.gov/ct2/show/record/ NCT00499330

5. Nakamura K, Saji H, Nakajima R, et al. A phase III randomized trial of lobectomy versus limited resection for small-sized peripheral non-small cell lung cancer (JCOG0802/WJOG4607L). Jpn J Clin Oncol 2010;40:271-4.

6. National Comprehensive Cancer Network. Non-Small Cell Lung Cancer. Version 01.2019. Available online: https://www.nccn.org/professionals/physician_gls/pdf/ nscl.pdf. Accessed on Nov 18, 2018.

7. Altorki NK, Kamel MK, Narula N, et al. Anatomical segmentectomy and wedge resections are associated with comparable outcomes for patients with small cT1N0 nonsmall cell lung cancer. J Thorac Oncol 2016;11:1984-92.

8. Nakamura H, Taniguchi Y, Miwa K, et al. Comparison of the surgical outcomes of thoracoscopic lobectomy, segmentectomy, and wedge resection for clinical stage I non-small cell lung cancer. Thorac Cardiovasc Surg 2011;59:137-41.

9. Martin-Ucar AE, Nakas A, Pilling JE, et al. A casematched study of anatomical segmentectomy versus lobectomy for stage I lung cancer in high-risk patients. Eur J Cardiothorac Surg 2005;27:675-9.

10. El-Sherif A, Gooding WE, Santos R, et al. Outcomes of sublobar resection versus lobectomy for stage I non-small cell lung cancer: a 13-year analysis. Ann Thorac Surg 2006;82:408-15; discussion 415-6.

11. Donahue JM, Morse CR, Wigle DA, et al. Oncologic efficacy of anatomic segmentectomy in stage IA lung cancer patients with T1a tumors. Ann Thorac Surg 2012;93:381-7; discussion 387-8.

12. Travis WD, Brambilla $E$, Noguchi $M$, et al. International Association for the Study of Lung Cancer/American Thoracic Society/European Respiratory Society International Multidisciplinary Classification of Lung Adenocarcinoma. J Thorac Oncol 2011;6:244-85.

13. Godoy MC, Truong MT, Sabloff B, et al. Subsolid pulmonary nodule management and lung adenocarcinoma classification: state of the art and future trends. Semin Roentgenol 2013;48:295-307.

14. Detterbeck FC, Boffa DJ, Kim AW, et al. The Eighth Edition Lung Cancer Stage Classification. Chest 
2017;151:193-203.

15. Goudie E, Oliveira RL, Thiffault V, et al. Phase 1 trial evaluating safety of pulmonary artery sealing with ultrasonic energy in VATS lobectomy. Ann Thorac Surg 2018;105:214-20.

16. Nomori H, Okada M. Illustrated Anatomical Segmentectomy for Lung Cancer. Springer Japan 2012.

17. Endoh M, Oizumi H, Kato H, et al. How to demarcate intersegmental plane with resected-segments inflation method using the slip knot technique in thoracoscopic anatomic segmentectomy. J Vis Surg 2017;3:100.

18. Iizuka S, Kuroda H, Yoshimura K, et al. Predictors of indocyanine green visualization during fluorescence imaging for segmental plane formation in thoracoscopic anatomical segmentectomy. J Thorac Dis 2016;8:985-91.

Cite this article as: Vieira A, Oliveira R, de Azevedo IS, Figueroa PU. Developing the guidelines: the techniques of uniportal VATS for sublobar resection and middle lobectomy. J Thorac Dis 2019;11(Suppl 16):S2086-S2094. doi: 10.21037/ jtd.2019.04.88 